\title{
Predictive Risk Factors for Early Seizures in Traumatic Brain Injury
}

\author{
Porntip Parmontree ${ }^{1}$ Thara Tunthanathip ${ }^{2}$ Thitima Doungngern ${ }^{1}$ Malee Rojpitbulstit ${ }^{1}$ \\ Wattanachai Kulviwat ${ }^{3}$ Sanguansin Ratanalert ${ }^{4}$
}

1 Faculty of Pharmaceutical Sciences, Prince of Songkla University, Songkhla, Thailand

2Department of Surgery, Division of Neurosurgery, Faculty of

Medicine, Songklanagarind Hospital, Prince of Songkla University, Songkhla, Thailand

${ }^{3}$ Department of Neurosurgery, Hatyai Hospital, Songkhla, Songkhla, Thailand

${ }^{4}$ School of Medicine, Mae Fah Luang University, Chiang Rai, Thailand

\begin{abstract}
Address for correspondence Thara Tunthanathip, Department of Surgery, Division of Neurosurgery, Songklanagarind Hospital, Prince of Songkla University, Hatyai, Songkhla 90110, Thailand (e-mail: tsus4@hotmail.com).
\end{abstract}

J Neurosci Rural Pract 2019;10:582-587

\begin{abstract}
Keywords

- posttraumatic seizure

- predictive model

- risk factors

- traumatic brain injury

Background Early posttraumatic seizure (PTS) is a significant cause of unfavorable outcomes in traumatic brain injury (TBI). This study was aimed to investigate the incidence and determine a predictive model for early PTS.

Materials and Methods A prospective cohort study of 484 TBI patients was conducted. All patients were evaluated for seizure activities within 7 days after the injury. Risk factors for early PTS were identified using univariate analysis. The candidate risk factors with $p<0.1$ were selected into multivariable logistic regression analysis to identify predictors of early PTS. The fitting model and the power of discrimination with the area under the receiver operating characteristic (AUROC) curve were demonstrated. The nomogram for prediction of early PTS was developed for individuals.

Results There were 27 patients (5.6\%) with early PTS in this study. The final model illustrated chronic alcohol use (odds ratio [OR]: 4.06, 95\% confidence interval [Cl]: 1.64-10.07), epidural hematoma (OR: 3.98, 95\% Cl: 1.70-9.33), and Glasgow Coma Scale score 3-8 (OR: 3.78, 95\% Cl: 1.53-9.35) as predictors of early PTS. The AUROC curve was 0.77 (95\% Cl: $0.66-0.87$ ).

Conclusions The significant predictors for early PTS were chronic alcohol use, epidural hematoma, and severe TBI. Our nomogram was considered as a reliable source for prediction.
\end{abstract}

\section{Introduction}

Traumatic brain injury (TBI) is a major health problem leading to mortality, disability, and decreased health-related quality of life. Severe TBI survivors may suffer with longterm disabilities and increased burden of health-care costs. Life expectancy of moderate-to-severe TBI is also reduced by 6.6 years. ${ }^{1}$ In the United States, it has been estimated that 3.2 to 5.3 million person live with a TBI-related disability. ${ }^{2}$

The major complication after TBI is the posttraumatic seizure (PTS) that can be classified into early PTS (occurring within 7 days) and late PTS (occurring after 7 days of injury). Early PTS may contribute to secondary brain injuries, including increased intracranial pressure, metabolic demand, and brain death. These are also crucial factor of late PTS.,4 Thus, the brain trauma foundation guidelines recommend the antiepileptic drug for early PTS prophylaxis. ${ }^{3,5}$ The incidence of early PTS varies from 4 to $25 \%$ and risk factors have been reported in the literatures. ${ }^{3,6-8}$

Temkin studied risk factors for PTS in 783 adults were surgery for severe head injury, operation of intracerebral hematoma, or subdural hematoma, ${ }^{7}$ whereas Ritter et al reported 
that early PTS increased in patients who underwent surgical evacuation operations. ${ }^{8}$ In addition, Wiedemayer et al using multivariate logistic regression analysis demonstrated that chronic alcohol abuse, subdural hematoma, and brain contusion were significant risk factors for early PTS and associated with unfavorable outcome. ${ }^{9}$ Therefore, predicting the risk factors for early PTS is necessary for proper management. Particularly, integrating predictive risk factors into an established tool is helpful for physicians to make clinical decisions. $^{10}$

Several investigators have described the risk factors for early PTS; however, the predictive risk factors have remained inconclusive. ${ }^{6,79,11}$ In Thailand, a few studies have reported the epidemiology of TBI, but none of them have studied the incidences and predictors for early PTS in adults. ${ }^{12,13}$ Since there is a lack of robust evidence, our study aimed to investigate the incidence and determine prognostic factors for early PTS. In addition, the nomogram for early PTS prediction was developed.

\section{Materials and Methods}

\section{Design}

This study was a prospective cohort in TBI patients who were admitted at Level I Trauma Centers in Southern Thailand from April 1, 2017, to March 31, 2018. The study was approved by the Institute Ethics Committee of Hatyai Hospital, and the Faculty of Medicine, Songklanagarind Hospital, Songkhla Province, Thailand.

\section{Participants}

Eligible participants were aged 15 years or older and diagnosed with TBI. Patients who had a history of TBI, seizures, brain tumor, stroke, or had undergone neurosurgical intervention were excluded from this study. Patients who were admitted to other facilities for $\geq 24$ hours before transfer to the study hospital were also excluded.

\section{Study Variables and Outcomes}

The data were obtained from direct patient or caregiver interviews, direct observation, and computerized medical records. The following variables were collected for baseline characteristics: age, gender, history of chronic alcohol use (daily use of alcohol for $\geq 1$ year), mechanism of injury and clinical presentation, Abbreviated Injury Scale score, Injury Severity Score, initial Glasgow Coma Scale (GCS) score, and types of neurosurgical procedures. Marshall computed tomography classification of TBI 14 was evaluated by the two neurosurgeons who participated in this study. The severity of TBI was categorized into three groups: mild TBI (GCS: 13-15), moderate TBI (GCS: 9-12), and severe TBI (GCS: $3-8$ ).

The outcome measurement of this study was clinical seizures or the presence of epileptic waveforms on electroencephalography (EEG) tests within 7 days after injury. Telephone monitoring for clinical seizures was performed if the patient was hospitalized for fewer than 7 days.

\section{Ethical Clearance}

The study was performed with permission from the Ethics Committee of the Faculty of Medicine, Songklanagarind Hospital, Prince of Songkla University (REC 60-059-19-9).

\section{Statistical Analysis}

The baseline characteristics were analyzed by the descriptive statistics, which were reported as the percentages for categorical data and mean \pm standard deviation (SD) for continuous data with a normal distribution. Seizure prevalence was calculated, and predictors were identified from the logistic regression model. Univariate regression analysis for the candidate factors, $p<0.1$, and clinical relevance were entered into the multivariable regression model. Furthermore, the intercorrelated variables with multicollinearity tolerance values of covariates $<0.5$ were examined. Backward multivariable regression was generated for prognostic models for early PTS based on Hosmer-Lemeshow goodness-of-fit test. To express the power of discrimination outcome, an area under the receiver operating characteristic (AUROC) curve was proposed and presented with $p>0.7$. Odds ratio (OR) with $95 \%$ confidence intervals, and $p<0.05$ was considered statistically significant. Statistical analyses were performed with the $\mathrm{R}$ version 3.4.0 software (R Foundation, Vienna, Austria).

After building the logistic regression model, the data were used to construct a clinical nomogram. 1000 bootstrapping was performed for internal validation of the nomogram. Therefore, the calibration plot was utilized to demonstrate how predictions of the nomogram compare with actual probabilities for calibration purpose. The R version 3.4.0 software with rms package (R Foundation, Vienna, Austria) was utilized for constructing the nomogram.

\section{Results}

Baseline characteristics are listed in -Table 1. A total of 484 patients were enrolled and $71.3 \%$ of them were male. The mean age of the patients was $43.1 \pm 19.3$ years. The common causes of injury were motorcycle crashes (62.2\%), falls (18.6\%), and body assault (5.4\%). The majority of patients had mild TBI (71.3\%). Computed tomography scan of the head showed subdural hematoma (30.8\%) and subarachnoid hemorrhage (27.5\%).

Twenty-seven patients (5.6\%) had early PTS. The majority of them was male (85.2\%), and two-third of cases was moderate-to-severe TBI. In addition, the mean age was 46.0 (SD: 18.5) years. The variables in univariate logistic regression are shown in - Table 2 . Seven candidate variables (chronic alcohol use, posttraumatic amnesia, subdural hematoma, epidural hematoma, size of midline shift, having undergone craniectomy, and initial GCS score) were selected into the multivariable logistic regression model. The minimum effect size for prediction was removed using backward multivariable logistic regression, and the remaining predictors, chronic alcohol use (OR: 4.06), epidural hematoma (OR: 3.98), and severe TBI (GCS: 3-8) group (OR: 3.78) were independently associated with early PTS in the multivariate 
Table 1 Characteristics of traumatic brain injury patients $(n=484)$

\begin{tabular}{|c|c|}
\hline Characteristics & $n(\%)$ \\
\hline Age (year), mean \pm SD & $43.1 \pm 19.3$ \\
\hline Male & $345(71.3)$ \\
\hline Chronic alcohol use & $50(11.1)$ \\
\hline \multicolumn{2}{|l|}{ Mechanism of injury } \\
\hline Motorcycle crash & $301(62.2)$ \\
\hline Fall & $90(18.6)$ \\
\hline Body assault & $26(5.4)$ \\
\hline Posttraumatic amnesia & $225(66.4)$ \\
\hline Loss of consciousness & $242(57.6)$ \\
\hline \multicolumn{2}{|l|}{ Type of injury } \\
\hline Blunt & $471(97.3)$ \\
\hline Penetrating & $10(2.1)$ \\
\hline Blunt and penetrating & $3(0.6)$ \\
\hline Intubation at the presentation & $150(31)$ \\
\hline \multicolumn{2}{|l|}{ Initial GCS score } \\
\hline 13-15 (mild TBI) & $345(71.3)$ \\
\hline 9-12 (moderate TBI) & $54(11.2)$ \\
\hline 3-8 (severe TBI) & 85 (17.5) \\
\hline \multicolumn{2}{|l|}{ ISS } \\
\hline$<16$ & $258(56.6)$ \\
\hline $16-24$ & $120(26.3)$ \\
\hline $25-75$ & $78(17.1)$ \\
\hline \multicolumn{2}{|l|}{ Head AIS score } \\
\hline $1-2$ & $200(44)$ \\
\hline 3 & $98(21.5)$ \\
\hline $4-5$ & $157(34.5)$ \\
\hline \multicolumn{2}{|l|}{ Marshall CT classification } \\
\hline Diffuse injury I & $178(36.9)$ \\
\hline Diffuse injury II & $150(31.1)$ \\
\hline Diffuse injury III & $90(18.7)$ \\
\hline Diffuse injury IV & $5(1.1)$ \\
\hline Diffuse injury $\mathrm{V}$ & $56(11.6)$ \\
\hline Diffuse injury VI & $3(0.6)$ \\
\hline \multicolumn{2}{|l|}{ CT brain finding } \\
\hline Subdural hematoma & $149(30.8)$ \\
\hline Subarachnoid hemorrhage & $133(27.5)$ \\
\hline Epidural hematoma & $73(15.1)$ \\
\hline Intracranial hemorrhage & $37(7.6)$ \\
\hline Midline shift $>5 \mathrm{~mm}$ & $26(40.6)$ \\
\hline Contusion & $83(17.2)$ \\
\hline Depressed skull fracture & $24(5.0)$ \\
\hline \multicolumn{2}{|l|}{ Neurosurgical procedure } \\
\hline Craniotomy & $41(8.5)$ \\
\hline Craniectomy & $31(6.4)$ \\
\hline
\end{tabular}

Abbreviations: AIS, Abbreviated Injury Scale; CT, computed tomography; ISS, injury severity score; GCS, Glasgow Coma Scale; SD, standard deviation; TBI, traumatic brain injury.
Table 2 Univariate analysis in traumatic brain injury patients with early posttraumatic seizure ( $n=27$ patients)

\begin{tabular}{|c|c|c|}
\hline Variables & OR (95\% Cl) & $p$-Value \\
\hline \multicolumn{3}{|l|}{ Chronic alcohol use } \\
\hline No & Reference & \multirow[t]{2}{*}{$<0.001$} \\
\hline Yes & $5.62(2.31-13.64)$ & \\
\hline \multicolumn{3}{|l|}{ PTA } \\
\hline No & Reference & \multirow[t]{2}{*}{0.064} \\
\hline Yes & $6.93(0.89-53.64)$ & \\
\hline \multicolumn{3}{|l|}{ Subdural hematoma } \\
\hline No & Reference & \multirow[t]{2}{*}{0.006} \\
\hline Yes & $3.01(1.37-6.61)$ & \\
\hline \multicolumn{3}{|l|}{ Epidural hematoma } \\
\hline No & Reference & \multirow[t]{2}{*}{$<0.001$} \\
\hline Yes & 4.38 (1.94-9.87) & \\
\hline \multicolumn{3}{|c|}{ Size of midline shift (mm) } \\
\hline$\leq 5$ & Reference & \multirow[t]{2}{*}{0.034} \\
\hline$>5$ & $3.43(1.09-10.81)$ & \\
\hline \multicolumn{3}{|l|}{ Craniectomy } \\
\hline No & Reference & \multirow[t]{2}{*}{0.013} \\
\hline Yes & $3.77(1.32-10.75)$ & \\
\hline \multicolumn{3}{|l|}{ Craniotomy } \\
\hline No & Reference & \multirow[t]{2}{*}{0.002} \\
\hline Yes & $4.35(1.72-11.02)$ & \\
\hline \multicolumn{3}{|l|}{ GCS score } \\
\hline 13-15 (mild TBI) & Reference & \\
\hline 9-12 (moderate TBI) & $3.09(1.03-9.30)$ & 0.044 \\
\hline 3-8 (severe TBI) & $4.51(1.88-10.80)$ & 0.001 \\
\hline \multicolumn{3}{|l|}{ Marshall CT classification } \\
\hline Diffuse injury I & Reference & \\
\hline Diffuse injury II & $2.13(0.61-7.42)$ & 0.235 \\
\hline Diffuse injury III & $2.02(0.49-8.28)$ & 0.327 \\
\hline Diffuse injury IV & $\mathrm{N} / \mathrm{A}$ & $N / A^{a}$ \\
\hline Diffuse injury $\mathrm{V}$ & $10.63(3.23-34.97)$ & $<0.001$ \\
\hline Diffuse injury VI & $21.75(1.62-291.97)$ & 0.02 \\
\hline \multicolumn{3}{|c|}{ Intubation at the presentation } \\
\hline No & Reference & \\
\hline Yes & $4.92(2.16-11.24)$ & $<0.001$ \\
\hline \multicolumn{3}{|l|}{ ISS } \\
\hline$<16$ & Reference & \\
\hline $16-24$ & $2.90(1.05-8.00)$ & 0.039 \\
\hline $25-75$ & $5.27(1.93-14.36)$ & 0.001 \\
\hline \multicolumn{3}{|l|}{ Head AIS score } \\
\hline $1-2$ & Reference & \\
\hline 3 & $0.81(0.15-4.26)$ & 0.806 \\
\hline $4-5$ & $5.37(1.95-14.72)$ & 0.001 \\
\hline
\end{tabular}

Abbreviations: AIS, Abbreviated Injury Scale; $\mathrm{Cl}$, confidence interval; $\mathrm{CT}$ computed tomography; GCS, Glasgow Coma Scale; ISS, injury severity score; OR, odds ratio; PTA, posttraumatic amnesia; TBI, traumatic brain injury. aExact confidence levels not possible with zero count cells. 
analysis ( - Table $\mathbf{3})$. The model was calibrated with the Hosmer-Lemeshow goodness-of-fit test $(p=0.495)$ and showed good discrimination through the AUROC curve of 0.77 .

After building the logistic regression models, the nomogram was constructed to predict the risk of early PTS of an

Table 3 Multivariable logistic regression model predicted risk factor of early posttraumatic seizure ( $n=27$ patients)

\begin{tabular}{|l|l|l|}
\hline Factor & Adjusted OR (95\% Cl) & $p$-Value \\
\hline Chronic alcoholism & $4.06(1.64-10.07)$ & 0.002 \\
\hline Epidural hematoma & $3.98(1.70-9.33)$ & 0.001 \\
\hline GCS score \\
\hline 9-12 (moderate TBI) & $2.63(0.84-8.24)$ & 0.097 \\
\hline 3-8 (severe TBI) & $3.78(1.53-9.35)$ & 0.004 \\
\hline
\end{tabular}

Abbreviations: $\mathrm{Cl}$, confidence interval; GCS, Glasgow Coma Scale; OR, odds ratio; TBI, traumatic brain injury. individual. Using the 1000 bootstrapping method, the points achieved from each parameter were summed, and the total points correspond to the risk of early PTS in percentages as shown in - Fig. 1. Therefore, the calibration plot was utilized for calibration purpose. In the calibration plot, the X-axis represents nomogram predictions and the Y-axis represents the observed rate of the outcome event in the validation cohort. The dashed $45^{\circ}$ line represents the ideal performance of a nomogram, in which the predicted outcome corresponds perfectly to the actual performance. Therefore, the concordance performance is presented as the bias-corrected calibration line in - Fig. 2.

\section{Discussion}

In this study, we developed the prognostic factors and investigated incidences through collecting data from Level I Trauma

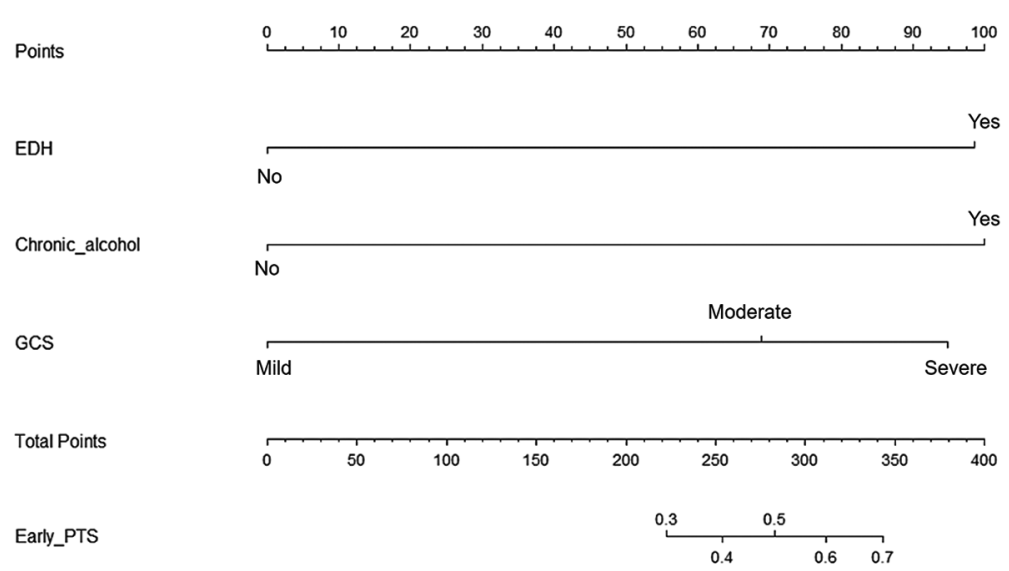

Fig. 1 Nomogram for predicting early posttraumatic seizure (PTS). To use the nomogram, draw a straight line upward from the patient's characteristics of Glasgow Coma Scale, epidural hematoma (EDH), chronic alcohol to the upper points scale, and the sum of the scores of all variables. Then, draw another straight line downward from the scale of the total points through the risk of early PTS. This is the probability of the presence of early PTS in an individual.

Fig. 2 Calibration plot.

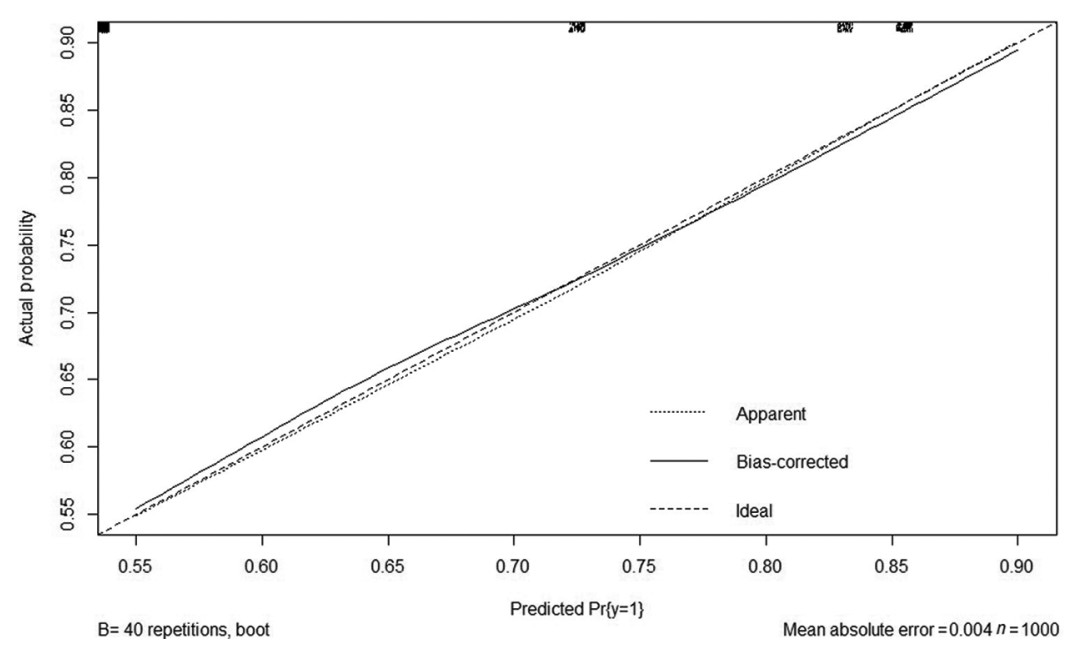


Centers in Southern Thailand. Our result reveals three factors which predict early PTS through the regression model. Furthermore, we constructed the nomogram from this model for predicting individual risk of early PTS in real-world clinical practice.

Early PTS observed in this study (5.6\%) was similar to the Wiedemayer et $\mathrm{al}^{9}$ study that conducted a retrospective study in Germany and found early PTS of 5.8\% in blunt head injury patients. When compared with the other previous studies, the incidence of early PTS was widely reported from 1.9 to $10.1 \%{ }^{14-20}$ The incidence of early PTS seemed to be higher in young children than in adults from prior studies, ${ }^{4,21}$ whereas the majority of patients with early PTS in the present study was found in adults.

Several researchers have examined the factors for PTS, 15-19,22,23 but few studies have introduced a predictive model for early PTS. ${ }^{9}$ After multivariate regression analysis, we found three predictive risk factors for early PTS that are chronic alcohol use, epidural hematoma, and severe TBI. Chronic alcohol use was previously described as a significant factor for early PTS. ${ }^{6,9,20}$ Similarly, our study revealed chronic alcohol use was the strongest predictor. The most common cause of traffic injury in Thailand is motorcycle crashes. ${ }^{12,24}$ Our study also found that motorcycle crashes were the leading cause of injury (62.2\%), and a history of alcohol use may be responsible for these injuries; as $78.9 \%$ of these patients declared that they had been drinking alcohol before involvement in a motorcycle accident. Alcohol consumption may increase seizure threshold by acting on gamma-aminobutyric acid receptors, while cessation of drinking may lower seizure threshold with upregulation of $\mathrm{N}$-methyl-D-aspartate receptor. Hence, in TBI patients, abrupt alcohol cessation may also trigger seizures, usually occurring within 6 to 48 hours of cessation..$^{25}$

Epidural hematoma was also a significant predictor of early PTS found in this study. Similar to the study of Temkin who reported that epidural hematoma increases the risk of early PTS by $17 \% .^{7}$ On the other hand, a subdural hematoma was shown to be a risk factor for early PTS in several studies. ${ }^{6,7,20}$ Based on our univariate analysis, both epidural hematoma and subdural hematoma were significant factors for early PTS with $p<0.001$ and 0.006 , respectively. However, when we inputted them into the multivariable model, only epidural hematoma was revealed as a strong factor for predicting early PTS.

The investigation of the association between TBI severity and early PTS has shown conflicting results. A few studies revealed the association between TBI severity and early PTS. The incidence of early PTS increased in patients with lower GCS scores. ${ }^{6,26}$ Our investigation found that in the multivariate analysis model, moderate-to-severe TBI (GCS <12) was a significant predictor for early PTS compared with mild TBI (GCS 13-15). In general, patients with low initial GCS scores have severe brain tissue damage that stimulates exchanges in extracellular ions and excessive release of glutamate, leading to enhanced excitatory connectivity. This mechanism relates to the stimulation of seizure activity. ${ }^{27}$ Thus, lower GCS scores showed an increased risk of seizure.
There are some limitations in this study. First, the incidence of PTS might be underdetected since data concerning clinical seizures were obtained from direct observation or self-reporting of seizure activity through telephone follow-up at day 7 after injury. Only $1 \%$ of the patients' seizure activity was monitored using EEG. A previous study reported that the epileptic waveform activities increased to $33 \%$ when continuous EEG monitoring was used. ${ }^{28}$ However, the use of EEG monitoring for seizure activity in TBI patients was limited in clinical practice. Clinical seizure observation has become the mainstay for seizure management. Second, this study was an observational study in real practice. We were unable to control the use of antiepileptic drugs for PTS prophylaxis resulting in a small number of patients with early PTS. Finally, although our analysis combined both close head injury and penetrated head injury, the majority of the patients (97\%) had blunt head injury. The utilization of this predictive model should be used with caution in cases of penetrating TBI.

Nevertheless, this study may have some strengths. To our knowledge, the present study is the first prognostic model for early PTS with a nomogram risk score. It could be used for early PTS prophylaxis management. Early prediction is important for controlling seizures and preventing secondary brain damage. Subsequently, we developed the nomogram for predicting this complication for an individual patient in real practice.

\section{Conclusions}

Our study provides the predictors for early PTS using multivariable analysis. The model indicates that chronic alcohol use, epidural hematoma, and severe TBI are strong predictors for early PTS. The use of this model for predicting the probability may be a useful management plan for seizure control in TBI.

\section{Ethical Approval}

All procedures performed in studies involving human participants were in accordance with the ethical standards of the institutional and/or national research committee and with the 1964 Helsinki declaration and its later amendments or comparable ethical standards.

\section{Funding}

The research funds were supported from the Faculty of Pharmaceutical Sciences and Graduate school, Prince of Songkla University, Thailand.

\section{Conflict of Interest}

None declared.

\section{Acknowledgments}

The authors would like to thank Naruemon Anumas, Prattana Chainiramol, and Supaporn Sainamsai for contributing to the data collection; Kanitha Arundon for being the coordinator of the research setting; and Maria Suzanne Mullet for English editing of the manuscript. 


\section{References}

1 Greenwald BD, Hammond FM, Harrison-Felix C. Nakase-Richardson R, Howe LL, Kreider S. Mortality following traumatic brain injury among individuals unable to follow commands at the time of rehabilitation admission: a national institute on disability and rehabilitation research traumatic brain injury model systems study. J Neurotrauma 2015;32(23):1883-1892

2 Centers for Disease Control and Prevention, Report to Congress Traumatic Brain Injury in the United States: Epidemiology and Rehabilitation. Atlanta, GA: Centers for Disease Control and Prevention, US Dept of National Center for Injury Prevention and Control; 2015

3 Bratton SL, Chestnut RM, Ghajar J, et al; Brain Trauma Foundation; American Association of Neurological Surgeons; Congress of Neurological Surgeonsjoint Section on Neurotrauma and Critical Care, AANS/CNS. Guidelines for the management of severe traumatic brain injury. XIII. Antiseizure prophylaxis. J Neurotrauma 2007;24(Suppl 1):S83-S86

4 Asikainen I, Kaste M, Sarna S. Early and late posttraumatic seizures in traumatic brain injury rehabilitation patients: brain injury factors causing late seizures and influence of seizures on long-term outcome. Epilepsia 1999;40(5):584-589

5 Carney N, Totten AM, O'Reilly C, et al. Guidelines for the management of severe traumatic brain injury, fourth edition. Neurosurgery 2017;80(1):6-15

6 Frey LC. Epidemiology of posttraumatic epilepsy: a critical review. Epilepsia 2003;44(s10):11-17

7 Temkin NR. Risk factors for posttraumatic seizures in adults. Epilepsia 2003;44(s10):18-20

8 Ritter AC, Wagner AK, Fabio A, et al. Incidence and risk factors of posttraumatic seizures following traumatic brain injury: a traumatic brain injury model systems study. Epilepsia 2016;57(12):1968-1977

9 Wiedemayer H, Triesch K, Schäfer H, Stolke D. Early seizures following non-penetrating traumatic brain injury in adults: risk factors and clinical significance. Brain Inj 2002;16(4):323-330

10 Chen F, Lin L, Yan L, et al. Nomograms and risk scores for predicting the risk of oral cancer in different sexes: a large-scale case-control study. J Cancer 2018;9(14):2543-2548

11 Chan KH, Tharakan J, Pal HK, Khan N, Tan YC. Risk factors and phenytoin prophylaxis for early post-traumatic seizures among patients with traumatic brain injury. Malays J Med Sci 2010;17(4):36-43

12 Phuenpathom N, Tiensuwan M, Ratanalert S, Saeheng S, Sripairojkul B. The changing pattern of head injury in Thailand. J Clin Neurosci 2000;7(3):223-225

13 Prathep S, Sriplung H, Phuenpathom N, Zunt J, Hirunpat S, Vavilala MS. Characteristics and outcomes of Thai patients hospitalized with severe traumatic brain injury between 2009 and 2011. J Med Assoc Thai 2017;100:843-849

14 Mata-Mbemba D, Mugikura S, Nakagawa A, et al. Early CT findings to predict early death in patients with traumatic brain injury: Marshall and Rotterdam CT scoring systems compared in the major academic tertiary care hospital in northeastern Japan. Acad Radiol 2014;21(5):605-611

15 Wang H, Xin T, Sun X, et al. Post-traumatic seizures-a prospective, multicenter, large case study after head injury in China. Epilepsy Res 2013;107(3):272-278

16 Thapa A, Chandra SP, Sinha S, Sreenivas V, Sharma BS, Tripathi M. Post-traumatic seizures-a prospective study from a tertiary level trauma center in a developing country. Seizure 2010;19(4):211-216

17 Najafi MR, Tabesh H, Hosseini H, Akbari M, Najafi MA. Early and late posttraumatic seizures following traumatic brain injury: a five-year follow-up survival study. Adv Biomed Res 2015;4:82

18 Englander J, Bushnik T, Duong TT, et al. Analyzing risk factors for late posttraumatic seizures: a prospective, multicenter investigation. Arch Phys Med Rehabil 2003;84(3):365-373

19 Rabiu TB, Adetunmbi B. Posttraumatic seizures in a rural Nigerian neurosurgical service. World Neurosurg 2017;104:367-371

20 Majidi S, Makke Y, Ewida A, Sianati B, Qureshi AI, Koubeissi MZ. Prevalence and risk factors for early seizure in patients with traumatic brain injury: analysis from national trauma data bank. Neurocrit Care 2017;27(1):90-95

21 Yablon SA. Posttraumatic seizures. Arch Phys Med Rehabil 1993;74(9):983-1001

22 Annegers JF, Hauser WA, Coan SP, Rocca WA. A population-based study of seizures after traumatic brain injuries. N Engl J Med 1998;338(1):20-24

23 Ritter AC, Wagner AK, Szaflarski JP, et al. Prognostic models for predicting posttraumatic seizures during acute hospitalization, and at 1 and 2 years following traumatic brain injury. Epilepsia 2016;57(9):1503-1514

24 Tunthanathip T, Phuenpathom N. Impact of road traffic injury to pediatric traumatic brain injury in Southern Thailand. J Neurosci Rural Pract 2017;8(4):601-608

25 Hillbom M, Pieninkeroinen I, Leone M. Seizures in alcoholdependent patients: epidemiology, pathophysiology and management. CNS Drugs 2003;17(14):1013-1030

26 Temkin NR, Dikmen SS, Wilensky AJ, Keihm J, Chabal S, Winn HR. A randomized, double-blind study of phenytoin for the prevention of post-traumatic seizures. N Engl J Med 1990;323(8):497-502

27 Ding K, Gupta PK, Diaz-Arrastia R. Epilepsy after traumatic brain injury. In: Laskowitz D, Grant G, eds. Translational Research in Traumatic Brain Injury. Boca Raton (FL): CRC Press/Taylor and Francis Group; 2016. Available from: https:// www.ncbi.nlm.nih.gov/books/NBK326725/. [Last accessed on 2018 Aug 07]

28 Ronne-Engstrom E, Winkler T. Continuous EEG monitoring in patients with traumatic brain injury reveals a high incidence of epileptiform activity. Acta Neurol Scand 2006;114(1):47-53 